\title{
Listeriosis cluster in Sydney linked to hospital food
}

\author{
Three patients were diagnosed with listeriosis in different hospitals within a short period. Rapid \\ molecular typing techniques and review of hospital menus using an electronic menu database allowed \\ prompt identification of the source of infection and implementation of control measures that prevented \\ further infections.
}

\section{Clinical record}

Between 4 and 12 April 2013, a public health unit in Sydney was notified of three patients in different tertiary hospitals in two local health districts (LHDs) who had tested positive for listeriosis. This unusual occurrence prompted concern that the cases might be linked through contaminated hospital meals because hospitals in these LHDs source food from the same suppliers. The public health unit led a public health investigation, which included representatives from the New South Wales Food Authority, OzFoodNet, two NSW reference laboratories, food services, dietetics services and hospital infection control staff, to determine whether there was a link between the cases.

\section{"Laboratory techniques ... were central to linking the affected patients with a [single] food item."}

Listeriosis is a notifiable disease in NSW, and cases are investigated in accordance with NSW Health control guidelines. ${ }^{1}$ All listeriosis notifications in NSW between 2 April (when the first case was detected) and 25 June (70 days after control measures were implemented) were reviewed in the process of case finding. A case was defined as a confirmed diagnosis of listeriosis during this period in any person in NSW who had been an inpatient of a public hospital in the two LHDs at any time during the potential exposure period for listeriosis; ie, 3-70 days prior to symptoms. Food history information was supplemented by records obtained from the electronic menu database (CBORD, Sydney) of the two LHDs, which records all hospital food menu items ordered by patients during their admissions. Positive blood cultures collected from patients underwent molecular subtyping, including binary typing, multiple-locus variablenumber tandem repeat analysis (MLVA), pulsed-field gel electrophoresis (PFGE) and molecular serotyping. Food and environmental specimens were screened for Listeria species, including L. monocytogenes, with a multiplex polymerase chain reaction (PCR) assay, and PCRpositive results were reported as presumptive positives and confirmed by culture; isolates from positive samples underwent molecular subtyping.

Sydney, NSW.

5 NSW Forensic and Analytical Science Services, Sydney, NSW.

zeina.najjar@ sswahs.nsw.gov.au

doi: 10.5694/mjal4.00913 blood cultures that tested positive for L. monocytogenes were collected on 6 April. Patient 3 was a 71-year-old with end-stage hepatocellular carcinoma and a history of two recent admissions to hospital C, and re-admitted on 8 April after a fall. Blood cultures collected that day were positive for L. monocytogenes. The patient died on 9 April.

Foods known to pose a risk of transmitting listeriosis, as well as food items consumed by all three patients during the overlapping periods of their admissions (20-26 March) were identified from hospital menus. Notable food items are listed in the Box.

The food safety profiles of the companies that manufactured these products were reviewed, and samples from local hospitals were tested on 16 April. There were no concerns about Companies Y and Z. Three weeks earlier, however, an environmental swab from the factory of Company X had tested positive for L. innocua during regular in-house testing, and a chocolate profiterole produced on 2 April had also tested positive for Listeria species; this batch had subsequently been discarded. Data from the menu database showed that all three patients had ordered chocolate profiteroles on 24 March. A leftover chocolate profiterole from the same batch was found in a local hospital and tested. The NSW Food Authority inspected the premises of Company $X$ on 17 April and collected food and environmental samples for testing.

On 18 April, the isolates from the three patients were reported as having identical binary types (223), MLVA profiles (04-17-16-05-03-11-14-00-16) and serotypes (1/2b, possible $3 b, 7)$, and on 15 May they were confirmed to share an identical PFGE pattern (4A:4:1). On 19 April, PCR testing of the chocolate profiterole left over from the batch ordered by the patients on 24 March returned

Foods with a high likelihood of transmitting listeriosis consumed by three Sydney patients diagnosed with listeriosis, by manufacturing company, 20-26 March 2013

Patient 1 Patient 2 Patient 3 Company

\begin{tabular}{|c|c|c|c|c|}
\hline $\begin{array}{l}\text { Creamy rice } \\
\text { pudding }\end{array}$ & $\checkmark$ & $\checkmark$ & $\checkmark$ & $Y$ \\
\hline $\begin{array}{l}\text { Chocolate } \\
\text { profiterole }\end{array}$ & $\checkmark$ & $\checkmark$ & $\checkmark$ & $x$ \\
\hline $\begin{array}{l}\text { Mango } \\
\text { cheesecake }\end{array}$ & $\checkmark$ & $\checkmark$ & & $x$ \\
\hline $\begin{array}{l}\text { Bread and } \\
\text { butter pudding }\end{array}$ & $\checkmark$ & & $\checkmark$ & $x$ \\
\hline $\begin{array}{l}\text { Sandwiches } \\
\text { (cold meats) }\end{array}$ & $\checkmark$ & $\checkmark$ & & Z \\
\hline
\end{tabular}


a presumptive positive result for L. monocytogenes, but repeat testing later indicated that this had been a false positive. All other samples of leftover hospital foods were negative for L. monocytogenes. Listeria was detected in seven environmental samples from Company X's premises, and L. monocytogenes was detected in a further two environmental samples from the production facility; the results of molecular subtyping of one of these samples (binary typing, MLVA and PFGE) were identical with those of the clinical specimens.

Creamy rice pudding was the first high-risk food identified and was temporarily withdrawn from all hospitals in the LHDs on 12 April until all products had been tested for L. monocytogenes. On 16 April, desserts from Company $X$ were withdrawn from all Sydney hospitals in which they were served. By the time of the presumptive positive PCR result, the risk of further infections in those who had consumed profiteroles was regarded as low, and it was decided not to proceed with the resource-intensive task of tracing all 1297 profiteroles served in hospitals within the LHDs on 24 March. Instead, active case finding was initiated by issuing a media release, alerting all treating doctors and general practitioners within the LHDs, and by establishing a public hotline number on 20 April.

\section{Discussion}

Listeriosis is an infection caused by L. monocytogenes and is transmitted through the ingestion of contaminated food, aided by the ability of the bacterium to survive some food processing techniques and to multiply at refrigerator temperatures. ${ }^{2,3}$ Listeriosis usually presents as non-invasive gastroenteritis in immunocompetent individuals, and as more severe invasive disease in older people, the immunocompromised and in pregnant women. ${ }^{2}$ Hospitalised patients are particularly likely to be older or immunocompromised, as were the patients in this cluster, and are therefore especially susceptible to listeriosis. ${ }^{4}$

Most cases of listeriosis are apparently sporadic, but foodborne outbreaks occur, and health care-associated listeriosis clusters have occasionally been reported. ${ }^{5,6}$ As the incubation period is quite long (3-70 days), it is often difficult to identify the vehicle of infection in listeriosis outbreaks. ${ }^{2,6}$ The increasing application and resolution of molecular subtyping of foodborne bacterial pathogens has, however, enabled investigators to detect clusters and track food sources of infection. ${ }^{7,8}$

Previous hospital outbreaks have not always identified specific vehicles of transmission, ${ }^{6}$ and inadequate records of the food items consumed by patients may have contributed to this failure. ${ }^{4}$ In the outbreak reported here, an electronic menu database enabled rapid identification of potential food sources and investigation of suppliers producing these foods, and prompt implementation of control measures. This would have been almost impossible with a manual menu system. The identification of this cluster led to the relevant services upgrading existing food safety plans to ensure that the future risk of hospitalacquired listeriosis is further minimised.
By identifying a rare and unique strain of L. monocytogenes in all three patients, the laboratory techniques used in this investigation were central to linking the affected patients with a food item produced by Company $X$. These techniques play an increasingly important role in detecting, investigating and controlling foodborne outbreaks. ${ }^{4,8-10}$ Binary typing and MLVA are PCR-based, and are the standard methods used in major Australian reference laboratories. Binary typing is rapid, with a turnaround time of 3 hours once DNA has been extracted from $L$. monocytogenes isolates. Binary type 223 is rare, and has been found in only one of 75 clinical L. monocytogenes food and environmental isolates in NSW since 2010, and in none of the 35 NSW isolates since 2012; its detection was the first indication of a link between the three patients in this report. MLVA has a greater discriminatory power than binary typing, with a turnaround time of 2-3 days; its use in parallel with binary typing was critical for the timely identification of a relationship between the isolates. PFGE is considered to be the gold standard of bacterial molecular typing because of its high discriminatory power. It is, however, time-consuming (requiring 4-5 days), technically demanding and not as portable as PCR-based typing methods. In this outbreak, it provided the confirmatory link between the clinical and environmental isolates.

Chocolate profiteroles were initially identified as the likely vehicle of transmission based on presumptive positive PCR results. PCR methods provide more rapid results than culture-based methods, and this prompted a public alert identifying profiteroles as the contaminated food source. When the PCR result was later deemed a false positive, chocolate profiteroles could no longer be viewed as the definite source of infection. Three further pieces of evidence nevertheless justified the decision to temporarily remove the products of Company $\mathrm{X}$ from hospital menus: the matching molecular profiles of isolates from the patients and from environmental samples from Company X; the patients' food consumption histories; and the detection by Company $\mathrm{X}$ of Listeria species in a profiterole produced on 2 April. There were no further infections after this decision was taken.

Rapid molecular subtyping was combined with reviewing an electronic hospital menu database to provide timely microbiological and epidemiological evidence that the three patients in the reported cluster probably acquired their infections from a contaminated hospital dessert produced by Company $X$. The identification of a likely source of infection and the quickly implemented control measures probably prevented further cases. This cluster of infections highlights the need for vigilant regulation and approval processes for food suppliers who service hospital populations.

Acknowledgements: We thank the Microbiology Diagnostic Unit, Public Health Laboratory, Victoria, for the performance of confirmatory MLVA and PFGE typing, as well as molecular serotyping. We also thank infection control staff and hospital management from the three hospitals for their assistance with the investigation. Competing interests: No relevant disclosures.

Provenance: Not commissioned; externally peer reviewed.

References are available online at www.mja.com.au. 
1 NSW Health. Listeriosis. Sydney: NSW Health, 2012. http:// www.health.nsw.gov.au/Infectious/controlguideline/Pages/ listeriosis.aspx (accessed Jun 2014).

2 Allerberger F, Wagner M. Listeriosis: a resurgent foodborne infection. Clin Microbiol Infect 2010; 16: 16-23.

3 Centers for Disease Control and Prevention (CDC). Outbreak of invasive listeriosis associated with the consumption of hog head cheese - Louisiana, 2010. MMWR Morb Mortal Wkly Rep 2011; 60: 401-405.

4 Cokes C, France AM, Reddy V, et al. Serving high-risk foods in a high-risk setting: survey of hospital food service practices after an outbreak of listeriosis in a hospital. Infect Control Hosp Epidemiol 2011; 32: 380-386.

5 Graham JC, Lanser S, Bignardi G, et al. Hospital-acquired listeriosis. J Hosp Infect 2002; 51: 136-139.

6 Gaul LK, Farag NH, Shim T, et al. Hospital-acquired listeriosis outbreak caused by contaminated diced celery - Texas, 2010. Clin Infect Dis 2013; 56: 20-26.

7 Hogg G, Tan A, Gregory J. Listeria surveillance in Australia from the laboratory perspective. Microbiol Aust 2013; 34: 90-92.

8 Allerberger F. Molecular typing in public health laboratories: from an academic indulgence to an infection control imperative. J Prev Med Public Health 2012; 45: 1-7.

9 Gilmour MW, Graham M, van Domselaar G, et al. High-throughput genome sequencing of two Listeria monocytogenes clinical isolates during a large foodborne outbreak. BMC Genomics 2010; 11: 120.

10 Sauders BD, Fortes ED, Morse DL, et al. Molecular subtyping to detect human listeriosis clusters. Emerg Infect Dis 2003; 9: 672-680. 